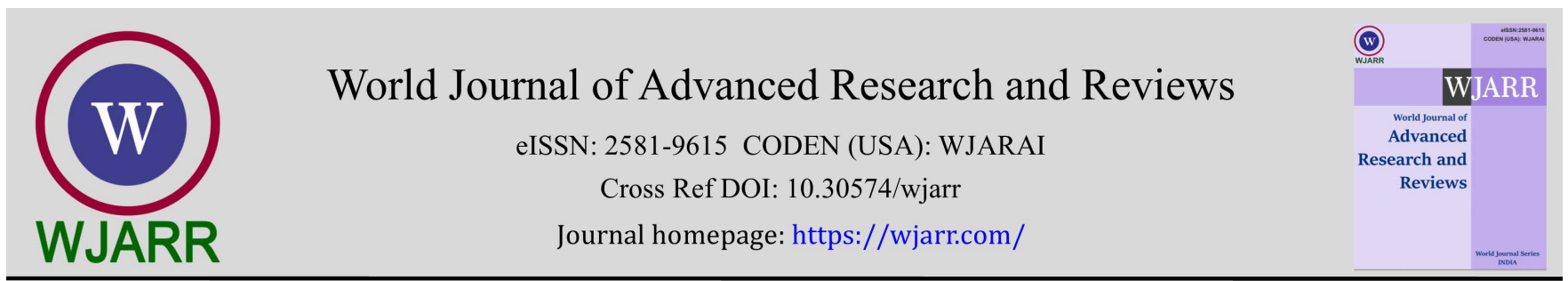

(REview ARTICLE)

Check for updates

\title{
The consequences of failed HIV prevention strategies: The ongoing challenges for societies
}

\author{
Reinhard H Dennin $1,{ }^{*}$ and Arndt Sinn 2 \\ ${ }^{1}$ Department of Infectious Diseases and Microbiology; University of Luebeck, UKSH, Campus Luebeck, Germany. \\ ${ }^{2}$ Centre for European and International Criminal Law Studies (ZEIS), University of Osnabrueck, Osnabrueck, Germany.
}

World Journal of Advanced Research and Reviews, 2021, 11(03), 391-398

Publication history: Received on 18 August 2021; revised on 19 September 2021; accepted on 21 September 2021

Article DOI: https://doi.org/10.30574/wjarr.2021.11.3.0469

\begin{abstract}
The spread of HIV in Europe is still ongoing. The established HIV prevention strategies base on structured goals to promote a behavior preventing HIV transmission, complemented by the provision of antiretroviral treatment. If followed by those people concerned, these multifactorial prevention strategies should encourage individual behavior that avoids HIV transmission in various ways, supplemented by medical help for people who are already HIV-infected.

Both national and international institutions have pointed to those who present late after being infected with HIV, the undiagnosed late presenters - who unknowingly may contribute to the spread of HIV. There are already initiatives for adjustments to the prevention strategies. In this context, reference is made to the burdens on societies.

In addition to the established prevention strategies, we propose the introduction of contact tracing. This measure could help reach and support people of the officially defined key populations to obey the prevention measures. This array might have a synergetic effect to contain the spread of HIV, thereby reducing the burden for societies.

A juridical analysis clarifies the handling of the term discrimination. Discrimination begins where a person is penalized because they are infected or ill. However, anyone who intentionally or negligently transmits HIV to unknowing people damages a person's health and this person is therefore also held criminally responsible in all legal systems. The socalled anti-discrimination concepts in the field of criminal law must not lose sight of this distinction.
\end{abstract}

Keywords: HIV infection; Prevention strategy; Behavior; Burden for societies

\section{Introduction}

- The messages of the HIV prevention strategies base on liberal concepts, the right of self-determination of those affected remains retained. However, the expectation of effectively containing the spread of HIV has not been fulfilled. An essential cofactor has turned out to be a crucial one for curtailing the ongoing spread of HIV, the unknowingly HIV infected people, the late presenters.

- Two currently overlapping pandemics caused by different viruses - a comparative analysis

SARS-CoV-2 is predominantly an airborne infection; [1] as viremic episodes occur, blood-borne transmission is possible. [2] Immunological naive persons in a community can become infected unnoticed by Sars-CoV-2. With time, this virus can become widespread. A protective vaccine against the original SARS-CoV-2 virus is available with a limited duration of effectiveness; additional measures including warning apps enabling contact tracing (CT) support assumptions that

\footnotetext{
${ }^{*}$ Corresponding author: Reinhard H Dennin

Department of Infectious Diseases and Microbiology; University of Luebeck, UKSH, Campus Luebeck, Germany.

Copyright (C) 2021 Author(s) retain the copyright of this article. This article is published under the terms of the Creative Commons Attribution Liscense 4.0.
} 
the COVID-19 pandemic can be limited to a low level in the long run. However, at present too many people ignore the prevention messages [3]. It's a serious constellation especially in the context of the "variants of concern" (VOC). This phenomenon is worrying because of the increasing prevalence of the disease burdens on societies as well as the various side effects. There are reports that the individual threat posed by SARS-CoV-2 could reduce the vigilance of people against HIV.

In contrast to SARS-CoV-2, HIV is essentially viremic, its infection is limited to a person-to-person contact and presents spotted distributions [4]; if no antiretroviral treatment (ART) is taken, progression to the end-stage AIDS occurs with high death rates. Who is mostly affected by HIV? The World Health Organization (WHO) has provided details for communities most affected by HIV transmission [5]. The WHO has designated key, limited populations who are at increased risk for HIV infection in all countries and regions. Key populations include "men who have sex with men; people who inject drugs; people in prisons; sex workers; transgender people" [6a], in addition to other vulnerable groups depending on the geographic areas. "Sexual transmission between men was the most common mode in the EU/EEA [European Union/European Economic Area], while heterosexual transmission and injecting drug use were the main reported transmission modes in the east of the Region" [7]. Potential HIV vaccines are currently being investigated. However, a setback has been reported. Various sources report that as a result of the contact restrictions due to the SARS$\mathrm{CoV}-2$ pandemic / epidemics, the demand for the pre-exposure prophylaxis (PrEP) to protect against the HIV infection has decreased.

\section{Main Article}

\subsection{The Key populations}

In the context of HIV prevention strategies, certain characteristics of these key populations need to be considered. They represent an inhomogeneous composition. Broadly speaking, they belong to multiple populations of different levels of education and social standards. Those with a low level of education and limited awareness are less able to understand and realize the prevention messages. In addition, their perception of risk varies depending on different motivations, including loss of control in situations where preventive measures are taken and age. With only a few exceptions, HIV is endemic in these key populations.

There is an issue that runs counter to the existing HIV prevention strategies because it indirectly promotes the spread of HIV: The incubation period for HIV infection can last up to several years. Late presenters might not know about their HIV infection until an HIV-positive diagnosis is made, but before the diagnosis they may have unknowingly infected more people with HIV. No medical care can be offered; they cannot benefit from early treatment with lifelong antiretroviral treatment (ART) [8].

\subsection{Facts About the HIV Pandemic and Epidemics in Selected Geographic Areas}

From the global perspective, there were "an estimated 38.0 million people living with HIV at the end of 2019; in Europe (the WHO European Region), [in] 2019, 2.6 million people were living with HIV" [9]. In 2019, there were 136,449 newly diagnosed HIV infections in the European Region, in 47 of the 53 Member States. The numbers were different in other geographic areas, including 24,801 from countries of the EU/EEA [10]. The number of people unaware of their HIV infection is estimated to 572,000 in the WHO European Region. Due to the multi-year HIV incubation period, many of them present with a late diagnosis. In 2019, about half (53\%) of those diagnosed with HIV in the European Region were diagnosed at a late stage of infection [11]. For the EU/EEA, in 2015 there were an estimated 122,000 people living with undiagnosed HIV, unaware of their HIV infection [12]. Until they are diagnosed as HIV-positive years after infection, they may have unknowingly spread HIV.

This situation indicates the need for countries to explore ways of increasing testing access and uptake [13].

For the United States, "At year-end 2019 there were approximately 1.2 million people in the U.S. have HIV. About 13 percent of them don't know it and need testing." [14 a] "HIV has cost America too much for too long and remains a significant public health issue." [14 b]

In their current forms, the key populations susceptible to HIV already pose burdens on societies, and there are more to come. Some reasons for these burdens are, among others, occult imponderables, such as people unaware of their HIV infection; people diagnosed as HIV positive, but without access to treatment; and people on ART but lacking sufficient suppression of the HIV load in blood, therefore being infectious to others. 


\subsection{HIV Prevention Strategies in Europe}

The WHO states that "The strategy promotes a people-centered approach, grounded in principles of human rights and health equity" [15]. The people at risk - the key populations - should also be committed to the "Duties to the Community" outlined in Article 29 of the Human Rights Declaration. The European Centre for Disease and Prevention Control (ECDC)/WHO published a clear message: "Although HIV infection is preventable, significant HIV transmission continues across the WHO European Region." [16].

The European Action Plan [17], which can be considered a prevention strategy, has been applied by states in Europe to contain the HIV pandemic. It "is structured around four strategic directions: optimize HIV prevention, diagnosis, treatment and care outcomes." The strategic directions are synoptically grounded on enlightenment and prevention based on information, counseling, access to HIV testing and treatment, solidarity, anti-discrimination, recommendations for safe sex, and syringe services programs (SSPs). The messages of the HIV prevention campaigns are intended to have a sustainable influence on the behavior of the key populations, such as avoiding risky sex and injecting drug use, which favors the person-to-person transmission of HIV. However, the underlying principles of the strategic directions do not contain any overarching components for their effective and ongoing enforcement.

These campaigns are accompanied by providing ART for all patients with HIV: the Treatment AS Prevention (TASP) approach. This regime includes the pre-exposure prophylaxis (PrEP) for those HIV-naïve people-who like to engage in situational, risky sexual engagements but want to be protected-and the post-exposure prophylaxis (PEP). However, there have been problems in the implementation and use of PrEP [18]; once infected with, HIV infection remains a chronic infection, ART just provides the reduction of the viral load. Such prospects can lead to people at risk of being inconsiderate during sex, i.e., not complying with both the correct messages of the prevention campaigns and adherence to ART. The TASP regime does not guarantee a suppressed viral load. Indeed, various circumstances result in an unsuppressed HIV load in the blood, and thus the person is infectious to other people. The various problems in the context of insufficient suppression of the HIV load have been analyzed by the ECDC [19]. The enforcement of HIV testing in the United States [20] might also benefit from CT (see section V). The question is: How to convince enough people with these campaigns? An initiative named "Power of Big Data" has provided tools for numerous information of different levels [21].

\subsection{Disenchantment at Two Levels}

The designers and political decision-makers are those responsible for drafting the liberal HIV prevention strategies. These entities have favored self-determination without associating mandatory restrictions to control the ongoing spread of HIV. They have believed that enough people can be convinced by the persuasiveness and the judiciousness of the prevention campaigns, including the access to treatment. They have expected those concerned to pursue cooperatively the messages of the prevention campaigns, supplemented by the availability of ART. They have expected that these endeavors would thereby curtail the spread of HIV, and mortality would decline as a result. However, their expectation that enough people from the key populations would behave responsibly and obey lasting protective behavior was a self-delusion!

Too many of the key populations have not obeyed the messages of the HIV prevention campaigns. The designers of the current prevention campaigns have not considered the following

- Human sexual behavior and drug addiction is complicated and guided by a wide range of emotions.

- The designers have misjudged the disposition-led behavior of too many affected people, both risk-prone HIV naïve and HIV infected ones.

- Too many of the affected people have failed to adjust their behavior in the sense of the prevention messages: Readiness and self-discipline are necessary to make decisions in the interests of one's own health and social responsibility. However, too many have negligently or deliberately refused to cooperate in these contexts.

- Too many of the people at risk of HIV do not understand that voluntary cooperation in HIV prevention is imperative.

- Discrimination and behavior: Many people of the general population deplore the misdemeanor of those of the key populations who promote the ongoing spread of HIV-despite and against the provided correct prevention measures! The behavior of those who continue risky lifestyles, who do not respect the integrity of other people's health, and who continue going against constitutional norms is not sanctioned; instead, it induces refusal and disapproval-but this is not discrimination (see details in the chapter Juridical Aspects). The rejection of behaviour that is dangerous to oneself or others does not constitute worse treatment or discrimination of people because of an infection or illness or their sexual orientation. The rejection refers to what a person does or does not do in the context of legal prohibitions and not to what a person is [22]. 
Given these facts, the prevention strategies applied have not reached enough vulnerable people to heed the messages thoroughly. The situation is analogous in the United States: "Addressing continued disparities will also be crucial, as data show that HIV prevention and treatment services are still not reaching the groups who could benefit most" [23]. A modified TASP strategy in the United Kingdom based on a strengthened "test and treat" regime and "scale up of PrEP" has shown effects in reducing the incidence of HIV [24].

In contrast to expectations, identifiable ongoing dynamics in the spread of HIV are facts. Therefore, it lacks framework conditions in the sense of corrective measures against misconduct. A grievous situation concerns the reservoir of people of the key populations and other vulnerable people, many of whom have not been reached by the HIV prevention strategies established; parts of them live unaware of their HIV infection! Since the beginning of the HIV epidemics, the strategies have lacked perspicacious components to uncover the unknowingly infected people. Depending on their lifestyles, these people contribute to a growing potential of unknowingly infected people, at least until they reach the advanced stage of immunosuppression that required them to seek medical advice - the late presenters - while spreading HIV and other sexually transmitted diseases (STD). They represent reservoirs replenishing the local HIV epidemics. The increasing pool of the unknowingly infected people has not been made a real thematic priority in the past.

\subsection{Adjustments are Required}

After around 35 years of HIV prevention strategies in countries that mainly follow liberal standards, supported by ART initiatives, the shortcomings to curtail the spread of HIV have resulted in disillusionments. The ECDC's World AIDS Day 2020 initiative, "What can be done?" might expect too much in terms of compliance of too many already-affected and risk-prone people [25]. The WHO stated: "HIV continues to be a public health issue" [6b].

After weighing different interests under the given circumstances - that is, both the people at risk and the obligations of governments to protect societies from the increasing pressures - it follows that an alternative should be considered given the multifactorial but inept prevention strategies. In particular, the prevention strategies have insufficiently focusing on reaching a preponderant part of key populations. The respective concepts must include an allocative function regarding the various patterns of uncooperative behavior of the key populations. We propose combining the established and adjusted prevention strategies with measures such as CT, allowing back-tracing chains of HIV. Situation related CT should be implemented in already established private as well as official social infrastructures with a focus on AIDS care services. Concomitant issues must make key populations feel like they are cooperating partners of the prevention campaigns - not opponents - so that they may gain positive perspectives.

\subsection{Achievable Effects for the Benefit of Those Affected}

CT measures through face-to-face contact eliminates any kind of possible discrimination. The contacting persons should be seen as partners. This approach could help in several ways:

- Identifying some, but not all, HIV-infected people who do not know about their HIV infection, that is, the unknowingly infected people.

- Retracing HIV infection chains by personal contacts would help to identify people earlier during their infection, thereby reducing the proportion of late presenters.

- With earlier detection, there would be fewer long-term effects on their health and, ultimately, less follow-up costs. For the present time, the late presenters make up to $53 \%$ of new positive diagnostics in the EU/EEA [11].

- People who have been diagnosed as HIV positive and have received ART, but who have been lost to follow-up for unknown reasons, could again be identified and start ART again.

- CT could help to detect and to interrupt at least partly chains of HIV infections, thereby reducing the replenishment of HIV-infected people.

- People conceivably with an unknown HIV infection could be offered an HIV test as a matter of routine. By overcoming language barriers, HIV-infected migrants could be tracked down and won over to the use of ART.

- Individual counseling could be offered for those who are either still HIV naïve or HIV infected but who consciously prefer high risks, for example, bare-backing. Psychologists familiar with the complex behaviors in the context of HIV could personally contact such vulnerable individuals and connect them with experienced consultants to help them.

- CT management could also help to provide medical care assistance, for example, depending on the status of the HIV infection, the diagnosis of comorbidities these people who may already be dealing with other diseases such as tuberculosis, syphilis, and Pneumocystis carinii (PC). It also includes conditions that HIV can establish in the central nervous system (CNS), inducing long-term disorders such as HIV-associated neurocognitive disorders (HAND) [26]. Even patients receiving ART may experience HIV-associated disorders of the CNS [27]. 
- Any person detected as HIV positive via CT could experience advantages to their treatment and health. All people addressed here could be helped by professional counseling concerning risk reduction, avoiding social dislocation or exclusion, and they could be integrated into medical care services.

\subsection{The Burdens for Societies}

HIV infection contributes direct and indirect costs. This concerns therapy for HIV-infected people, and societies will be confronted for some decades with other services. Hence, HIV remains a major public health concern - apart from possible pressures to socioeconomic stability - as a result of the liberal prevention strategies.

Some exemplary perspectives regarding the burdens HIV presents to societies are presented below.

- The costs for ART are lifelong, ranging from children to adolescents -based on current knowledge - because ART does not provide a cure.

- Financial support for those in need is provided by cost bearers such as health insurance companies, health care services, governments, funders (e.g., global funder organizations), and civil care organizations.

- There are costs for treatments of the long-term somatic or mental secondary diseases due to HIV infection, including ambulatory care and hospitalization.

- Services are required for the unemployed as a result of their HIV infection, including payments for early retirement.

- There are costs for new initiatives, especially to train peer-workers to reach the "unknowingly infected" people, ranging from helpless situations to those intentionally rejecting compliance.

- There is an unknown number of challenges, especially the variety of financial burdens that will affect societies for several decades.

- Even if a preventive HIV vaccine should become available, the burden on societies from those currently living on lifelong TASP will continue for some decades.

\subsection{Juridical Aspects}

There is probably no state in the world where intentional or negligent bodily injury or homicide is not punishable. Rather, it is one of the basic pillars of criminal law that injuries to life and limb of other people require a reaction. The way in which this injury or killing happens is usually not described concretely by the legal systems. Rather, it is sufficient that an injury or killing is based on the behavior of a person and that this behavior can be attributed to the person as intentional or negligent. When the state describes such behavior as punishable, it thereby enters into an obligation: protection of physical integrity and life.

For the intentional or negligent killing or bodily injury caused by transmission of HIV, or an HIV-infected person who is aware of their illness and who wants to cause death, the general criminal offences therefore also apply in Germany. This follows an abstract concept for the protection of life and physical integrity without punishing a special offense in which the manner of injury to another person is specifically described by HIV transmission.

Criminality begins where another person intentionally or negligently kills or injures, or intentionally attempts to kill or injures another person by HIV transmission. However, this abstract protection concept poses certain challenges for criminal prosecution because it is scientifically not easy to prove the connection (causality) between the act of injury (HIV transmission) and the illness of the infected person due to the high variability of the HIV genome.

Even if it is not possible to prove causality in a specific case or if there was no infection, an attempted offense (bodily harm, manslaughter) can still be considered if the offense was committed intentionally. The focus of such a consideration is then the intent to commit the offense. This must include the fact that the person is aware of their own infection and therefore has the knowledge that they can transmit HIV. In this context, ART drugs play a major role. For if the person knows that their own viral load is so reduced that transmission of HIV is an unlikely event, knowledge of their own potential danger will be lacking. At the very least, however, this has an impact on intent (see below). If there is a clear lack of knowledge because, for example, no testing was carried out, criminal conduct is also excluded if HIV was not transmitted. Second, if a person has knowledge of their own potential danger, they must also have wanted to injure or to cause the death of the other person. This is called the voluntative element of intent. Here, in the German context, it is a question of whether someone approves of the death or injury. Specifically, it means whether the person, knowing the possible consequences of their own behaviour, has resigned himself to these potential consequences or is indifferent to them. In this context, protective mechanisms play a role, because a person who uses condoms during sexual intercourse, for example, does not want to injure or to cause the death of their partner in the legal sense, but tries 
to avoid it precisely through the protective measures. The person's own potential danger is thus reduced, a factor that has a direct effect on the voluntative element of intent.

Classical criminal law therefore does not have a discriminatory character with regard to people infected or ill with HIV, which is why it does not require any reforms due to the lack of an object of decriminalization. Against the background of the offenses to protect life and physical integrity, it is irrelevant whether another person is infected with HIV and therefore injured, or whether this person is deliberately infected with COVID-19 by coughing on another person. In many legal systems, the intentional or negligent transmission of HIV is dealt with using the classic instruments of criminal law, without the need to include the HIV phenomenon explicitly. Other legal systems are linked, for example, to unprotected sexual intercourse without informing the partner about one's own infection. Apart from the fact that these legal systems could also work with the classic criminal offenses, there are no fewer practical problems. Because if one links it to the knowledge of one's own infection, this leads to people no longer getting tested. In addition, it remains unexplained-and this is seen as a discriminatory aspect-why other diseases are not also covered. To solve this problem, one would have to link to the risk potential of all STD, as some jurisdictions have done. The very different legislation in the individual states of the United States shows very clearly the range of all criminal law options. Therefore, when discussing decriminalization in the context of HIV, it is also necessary to exclude traditional criminal law, because these instruments do not explicitly criminalize HIV.

We must be clear that the instruments of criminal law can only ever be the last means in a chain of measures to deal with an unwanted event. It can achieve something where the normative command, for example "do not hurt another person," is accepted and thus has a preventive effect in society. Criminal law cannot achieve anything where these messages do not arrive or are not accepted. In these cases, criminal law is only a blunt reaction to the offense: It no longer prevents anything, but only reacts to the breach of norms. However, this reaction is also important, because without the individual reaction, the norm violation would become a model for other people, and the validity of the norm would lose its power.

\subsection{Expectations}

Notwithstanding the declining trends in the spread of HIV that have been seen in certain geographic areas, at present there has not been a sufficient decline in new HIV infections despite the prevention campaigns, including the TASP strategy.

Where available, the liberal HIV prevention strategies tolerate lifestyles indirectly "allowing" the "key populations, vulnerable and other risk prone people" to counteract (a) the prevention messages and (b) the legal norms prescribed by constitutional defaults and social norms. However, the protection of human life cannot be a matter of contention. The expectation of thoroughly effective participation of a sufficient part of the relevant populations in line with the prevention messages has been inept since the beginning. Those responsible for the current HIV prevention strategies have been unable to assess the long-lasting causes and effects on societies, such as the steadily increasing consumption of health care resources.

The HIV/AIDS pandemic still reflects enduring challenges for societies. The setbacks have placed various kinds of burdens on societies for decades, and these burdens will continue into the foreseeable future. Despite the key strategies for HIV prevention, an expectation for the United States reads: "Without intervention nearly 400,000 more Americans will be newly diagnosed over 10 years despite the availability of tools to prevent transmissions" [14b]. The various "wake-up" calls (Chapters II and III) urge for adjustments of the current prevention strategies. Given the enduring spread of HIV, accompanying restrictive measures in this critical decade are required to cope with the spread of HIV [28]. Updated prevention strategies must contain tailored concepts targeting selfish gains, including the anti-social interests of those concerned - considering similar topics due to Covid-19. [29] Addressing programmatic measures in accordance with the constitutions of countries, such as avoiding infection with a mostly deadly outcome, are necessary.

The recent Political Declaration of the UNAIDS Executive Director Winnie Byanyima should be taken as a serious cautionary appeal in this context [30].

\section{Conclusion}

The spread of HIV continues, albeit with regional and geographical differences. The prevention strategies introduced by official institutions require those people assigned to the risk groups follow the relevant recommendations. However, too many people of the risk groups have abused this freedom of movement. This offense of the endangered has the 
consequence that the societies have incurred immense costs. Thanks to efficacious medical interventions - but no cure - against HIV infection, the burdens on societies will increase for several decades.

\section{Compliance with ethical standards}

\section{Acknowledgments}

Both authors have collaborated on the subject; there was no outside influence.

\section{Disclosure of conflict of interest}

Both authors have no conflicts of interest of any kind

\section{References}

[1] Lewis D. COVID-19 Rarely Spreads Through Surfaces. So Why Are We Still Deep Cleaning? Nature. 2021; 590: 2628.

[2] Peder L, Myhre, Christian Prebensen, , Christine Monceyron Jonassen, Jan Erik Berdal, Torbjørn Omland; SARSCoV-2 Viremia is Associated With Inflammatory, But Not Cardiovascular Biomarkers, in Patients Hospitalized for COVID-19; J American Heart Association. 2021; 10: e019756.

[3] Kondratenko T. Berlin Parties as Pandemic Threatens to Return. 2020.

[4] Center for Disease Control and Prevention (CDC), 27/4; Ways HIV can be transmitted. 2021.

[5] World Health Organization; HIV/AIDS. 2020.

[6] World Health Organization; a. GLOBAL HEALTH SECTOR STRATEGY ON HIV 2016-2021, TOWARDS ENDING AIDS, June 2016. b. HIV/AIDS, Key facts.

[7] European Centre for Disease Prevention and Control/World Health Organization; HIV/AIDS Surveillance in Europe 2019 - 2018 Data; Overview of HIV and AIDS in Europe. 2019.

[8] Jeffries WL IV, Dailey AF, Jin C, Carter JW, Scales L. Trends in Diagnosis of HIV Infection, Linkage to Medical Care, and Viral Suppression Among Men Who Have Sex with Men, by Race/Ethnicity and Age - 33 Jurisdictions, United States, 2014-2018. Morbidity and Mortality Weekly. 2021; 69: 1338-1342.

[9] World Health Organization; Latest HIV Estimates And Updates on HIV Policies Uptake. 2020.

[10] European Centre for Disease Prevention and Control/World Health Organization; HIV/AIDS Surveillance in Europe, 2020, 2019 Data; Overview of HIV and AIDS in Europe. 2020.

[11] World Health Organization; HIV/AIDS. 2019.

[12] Pharris A, Quinten C, Noori T, Amato-Gauci A J, van Sighem A. ECDC HIV/AIDS Surveillance and Dublin Declaration Monitoring Networks; Estimating HIV incidence and number of undiagnosed individuals living with HIV in the European Union/European Economic Area, 2015. Euro Surveillance. 2016; 21(48): pii= 30417.

[13] European Centre for Disease Prevention and Control/World Health Organization; HIV/AIDS surveillance in Europe 2020, 2019 Data. 2020.

[14] Office of Infectious Disease and HIV/AIDS Policy; Overview: What Is Ending the HIV Epidemic in the U.S.? 2021.

[15] World Health Organization; Global Health Sector Strategy on HIV, 2016-2021; Towards ending AIDS. 2016.

[16] European Centre for Disease Prevention and Control/WHO Regional Office for Europe; HIV/AIDS Surveillance in Europe 2019 - 2018 Data. Overview of HIV and AIDS in Europe. 2019.

[17] World Health Organization; European Action Plan for HIV/AIDS, 2012-2015. 2011.

[18] Kumar S, Haderxhanaj LT, Spicknall IH. Reviewing PrEP's Effect on STI Incidence Among Men Who Have sex with Men-Balancing Increased STI Screening and Potential Behavioral Sexual Risk Compensation. AIDS and Behavior. $2021 ; 25: 1810-1818$.

[19] European Centre for Disease Prevention and Control; Evaluating HIV Treatment as Prevention in the European Context. 2012. 
[20] Levine RL. National HIV Testing Day 2021. 2021.

[21] Olatosi B, Vermund SH, Li X. Power of Big Data in ending HIV. AIDS, 2021; 35: S1-S5.

[22] McDermott A. Harmful social behaviors are the toughest to shift. 2021.

[23] Centers for Disease Control and Prevention; Information from CDC's Division of HIV/AIDS Prevention. 2021.

[24] Brizzi F, Birrell PJ, Kirwan P, Ogaz D, Brown AE, Delpech VC. Noel Gill O, De Angelis D. Tracking Elimination of HIV Transmission in Men Who Have Sex With Men in England: A Modelling Study. The Lancet HIV, 2021; 8: e440e448.

[25] European Centre for Disease Prevention and Control; World AIDS Day 2020: The Way Towards 2030: Diversified Testing to Diagnose HIV Early. 2020; https://www.ecdc.europa.eu/en/news-events/world-aids-day-2020towards-2030.

[26] Ellis RJ, Calero P, Stockin MD. HIV Infection and the Central Nervous System: A Primer. Neuropsychology Review. 2009; 2: 144-151.

[27] National Institute of Neurological Disorders and Stroke; Neurological Complications of HIV and AIDS Fact Sheet. 2019.

[28] Kalomparis A. One Europe to End the HIV/AIDS Epidemic. 2019.

[29] Krause PR, Fleming TR, Peto R, et al; Considerations in boosting COVID-19 vaccine immune responses. Published Online. 13 September 2021.

[30] Byanyima W. Opening remarks by UNAIDS Executive Director. June 2021. 